DOI: $10.24850 /$ j-tyca-2018-04-03

Artículo

\title{
Modelación hidrológica e hidráulica de un río intraurbano en una cuenca transfronteriza con el apoyo del análisis regional de frecuencias Hydrological and hydraulic modeling of an intra-urban river in a transboundary basin using a regional frequency analysis
}

Carlos Salazar-Briones ${ }^{1}$

Michelle Hallack-Alegría²

Alejandro Mungaray-Moctezuma ${ }^{3}$

Marcelo A. Lomelí ${ }^{4}$

Alvaro Lopez-Lambraño ${ }^{5}$

Adrián Salcedo-Peredia 6

${ }^{1}$ Universidad Autónoma de Baja California, Mexicali, Baja California, México, csalazar86@uabc.edu.mx

${ }^{21}$ Universidad Autónoma de Baja California, Mexicali, Baja California, México, mhallack@mtu.edu

3Universidad Autónoma de Baja California, Mexicali, Baja California, México, alejandro.mungaray@uabc.edu.mx

«Universidad Autónoma de Baja California, Mexicali, Baja California, México, Iomeli.marcelo@uabc.edu.mx

5Universidad Autónoma de Baja California, Mexicali, Baja California, México, alopezl@uabc.edu.mx

6Universidad Autónoma de Baja California, Mexicali, Baja California, México, salcedo.adrian@uabc.edu.mx

Autor para correspondencia: Carlos Salazar-Briones, csalazar86@uabc.edu.mx

\section{Resumen}

Las inundaciones se encuentran entre los peligros naturales más recurrentes y devastadores, al afectar vidas humanas y causar graves 
daños económicos en todo el mundo. Las regiones áridas y semiáridas son particularmente vulnerables a tormentas intensas en periodos cortos debido a que provocan inundaciones súbitas. Estas regiones representan un $30 \%$ del área mundial y están habitadas por $20 \%$ de la población. El objetivo principal del presente estudio es estimar la tormenta de diseño para los periodos de retorno de 20, 50, 100 y 500 años en la región semiárida de la subcuenca del Río Nuevo, a fin de determinar las áreas de inundación del cauce principal. Se propone un modelo integrado, que consiste en desarrollar un acoplamiento del modelo hidrológico e hidráulico para diferentes periodos de retorno, alimentados con un Análisis Regional de Frecuencia (ARF), utilizando el enfoque de los Lmomentos, empleando los programas HEC-HMS y HEC-RAS. Las áreas de inundación obtenidas de 190.55 a 237.83 ha y profundidades desde 0.10 hasta 6.0 metros comprometen la infraestructura urbana de la ciudad. Los resultados de esta investigación pueden ser utilizados por organismos encargados de la planeación urbana para disminuir riesgos de inundación.

Palabras clave: inundación, tormenta de diseño, análisis regional de frecuencia, L-momentos, HEC-HMS, HEC-RAS, sistema de información geográfica, simulación.

\section{Abstract}

Floods are among the most recurrent and devastating natural hazards, affecting human lives and causing severe economic damage worldwide. Arid and semi-arid regions are particularly vulnerable to intense storm events over short time periods resulting in flashfloods. These regions represent approximately $30 \%$ of the world's area and are inhabited by approximately $20 \%$ of the total population. The main objective of this study was to estimate the design storm for return periods of 20,50,100, and 500 years, in the semi-arid region of the New River sub-basin, in order to determine the flood areas in the main channel. In this study, an integrated model approach was proposed, consisting of developing a hydrological model for different return periods and performing a regional frequency analysis (RFA) using the L-Moments approach, in order to generate the input for hydrological and hydraulic modeling using the programs HEC-HMS and HEC-RAS. The flood areas obtained ranged from 190.55 ha to 237.83 ha, and depths from 0.10 to 6 meters, which pose a risk to the urban infrastructure of the city. The outcome of this research provides information that can be used by urban planners to reduce flood risks.

Keywords: Flood areas, design storm, regional frequency analysis, Lmoments, HEC-HMS, HEC-RAS, SIG, simulation. 
Recibido: 20/10/2017

Aceptado: $14 / 02 / 2018$

\section{Introducción}

Se estima que alrededor de 300 millones de personas han sido afectadas desde la década de 1990 por desastres naturales (Trivedi, Singh, \& Indian, 2015). Según el Grupo Intergubernamental de Expertos sobre el Cambio Climático (IPCC, por sus siglas en inglés) se han observado desviaciones en los patrones meteorológicos promedio del mundo, con una mayor frecuencia de eventos extremos de precipitación (UNHABITAT, 2011). En este sentido, las inundaciones se encuentran entre los peligros naturales más recurrentes y devastadores (Ouma \& Tateishi, 2014). Las inundaciones corresponden a $50 \%$ de los desastres relacionados con el agua, por encima de hambrunas, sequías y epidemias (Hernández-Uribe, Barrios-Piña, \& Ramírez, 2017).

La aceleración del crecimiento poblacional y los cambios en el uso de suelo aumentan la vulnerabilidad ante las inundaciones, incluyendo mortalidad y morbilidad, desplazamiento, y daño a cultivos, así como a la infraestructura y propiedad (Dewan, 2015). Existe una relación directa entre urbanización y cambios en las características hidrológicas, incluyendo disminución de la infiltración, aumento de escorrentía y altura de las inundaciones que, aunado al crecimiento de la población y a la acumulación de activos de valor, agravan el riesgo de inundación en zonas urbanas (Ouma \& Tateishi, 2014). Por lo anterior, las cuencas urbanas resultan más vulnerables a estos fenómenos, sobre todo por los procesos de origen antrópico propios de su dinámica (UN-HABITAT, 2011).

La principal característica de la precipitación en zonas áridas y semiáridas consiste en tormentas que se intensifican con rapidez, provocando inundaciones repentinas (Pourreza, Samadi, Akhoond, \& Ghahraman, 2016). En estas regiones habita alrededor de $20 \%$ de la población total y representan un 30\% del área mundial (Sivakumar, Das, \& Brunini, 2005).

Este estudio se realiza en el Río Nuevo, dentro de la cuenca transfronteriza del Salton Sea, ubicada en la región semiárida del Delta del río Colorado, colindante con la frontera entre los estados de Baja California, México, y California, Estados Unidos (The Salton Sea Authority, 2015). El Río Nuevo es un componente intraurbano, ubicado en la ciudad de Mexicali, Baja California, México, que conduce, además de 
escurrimientos superficiales derivados de eventos locales por precipitación, aguas residuales de origen agrícola, aguas provenientes de procesos industriales y aguas negras.

La importancia del estudio de eventos extremos de precipitación por tormentas de alta intensidad y corta duración en zonas áridas y semiáridas estriba en la capacidad de daños que estos fenómenos pueden ocasionar en la población, y en la infraestructura urbana y agrícola, así como en las actividades de importancia socioeconómica. En el caso del condado Imperial, en California, Estados Unidos, a partir de 1958 y hasta 2006 se habían declarado cuatro estados de emergencias debido a inundaciones, presentando severos daños en la infraestructura hidráulica, agrícola y carretera (Imperial County, 2013). La situación en Mexicali es distinta, pues la zona con mayor riesgo de inundación se presenta en su núcleo urbano, en particular en el Río Nuevo, donde se ha desarrollado infraestructura educativa, comercial, de gobierno y asentamientos humanos.

Tomando como referencia lo anterior y entendiendo que en la evaluación del riesgo de inundación es importante una base científica para la gestión del riesgo de desastres, el objetivo de este estudio es determinar las áreas de inundación del cauce principal del Río Nuevo, caracterizando una tormenta de diseño en condiciones estándar, y con periodos de retorno de 20, 50, 100 y 500 años, a través del acoplamiento de los modelos hidrológico e hidráulico. Esto incluye el empleo de un análisis regional de frecuencia (ARF), con enfoque en los L-momentos que, según Wallis, Shaefer, Barker y Taylor (2007), ha demostrado la fiabilidad de las estimaciones de magnitud de ocurrencia de precipitación.

Referente a los modelos hidrológicos, desde el desarrollo del método racional por Mulvany (1850), se inició la evolución de los mismos, pasando por modelos simples (concentrados) para eventos hasta el desarrollo de modelos distribuidos para simulaciones eventuales y continuas (Vargas-Castañeda, Ibáñez-Castillo, \& Arteaga-Ramírez, 2015). Los modelos usados para predecir etapas y flujos en ríos están bien adoptados en la práctica. La gama actual de métodos de modelado fluvial puede dividirse en tres grupos: 1 ) el proveniente de las ecuaciones de Saint-Venant, incluyendo HEC-RAS; 2) modelos basados en datos observados, donde la mayoría de los parámetros carece de una interpretación física directa, y 3 ) un grupo que se encuentra entre los dos anteriores e incluye modelos conceptuales que emanan de la ecuación de continuidad (Wolfs, Meert, \& Willems, 2015).

Para el análisis hidrológico de este estudio se optó por el empleo de un modelo semidistribuido, como lo es el Hydrologic Modelig System (HEC$H M S)$, mismo que ha sido ampliamente utilizado para evaluar efectos en los hidrogramas ante posibles escenarios de cambio climático, tipo de uso 
de suelo, diseño y gestión de obras hidráulicas, así como en la delimitación de zonas inundables (López et al., 2012). Por otro lado, la simulación hidráulica se realizó mediante el software River Analysis System (HEC-RAS), que cuenta con una serie de componentes de análisis unidimensional y bidimensional de flujo en ríos que permite simular el flujo de agua en un modelo 2D, a fin de determinar la llanura de inundación.

Según el estado del arte referente a la modelación hidrológica e hidráulica, la mayoría se realiza de forma independiente, limitando la capacidad de los modelos para pronosticar niveles de inundación debido a errores ocasionados por la manipulación y transferencia de la información (Rodríguez, González, Medina, Pardo, \& Santos, 2007). Así, este estudio propone un modelo integral a través de la puesta en marcha de un modelo hidrológico, alimentado con los resultados del ARF, con el enfoque de los $L$-momentos, y su acoplamiento con un modelo hidráulico, empleando las herramientas de modelado de HEC-HMS y HEC-RAS.

\section{Área de estudio}

El Río Nuevo se encuentra en una cuenca transfronteriza al noroeste de México, constituida por la participación de distintos núcleos urbanos. Al norte colinda con el condado Imperial, en California, Estados Unidos; y al sur, con la ciudad de Mexicali, Baja California, México (Figura 1). 

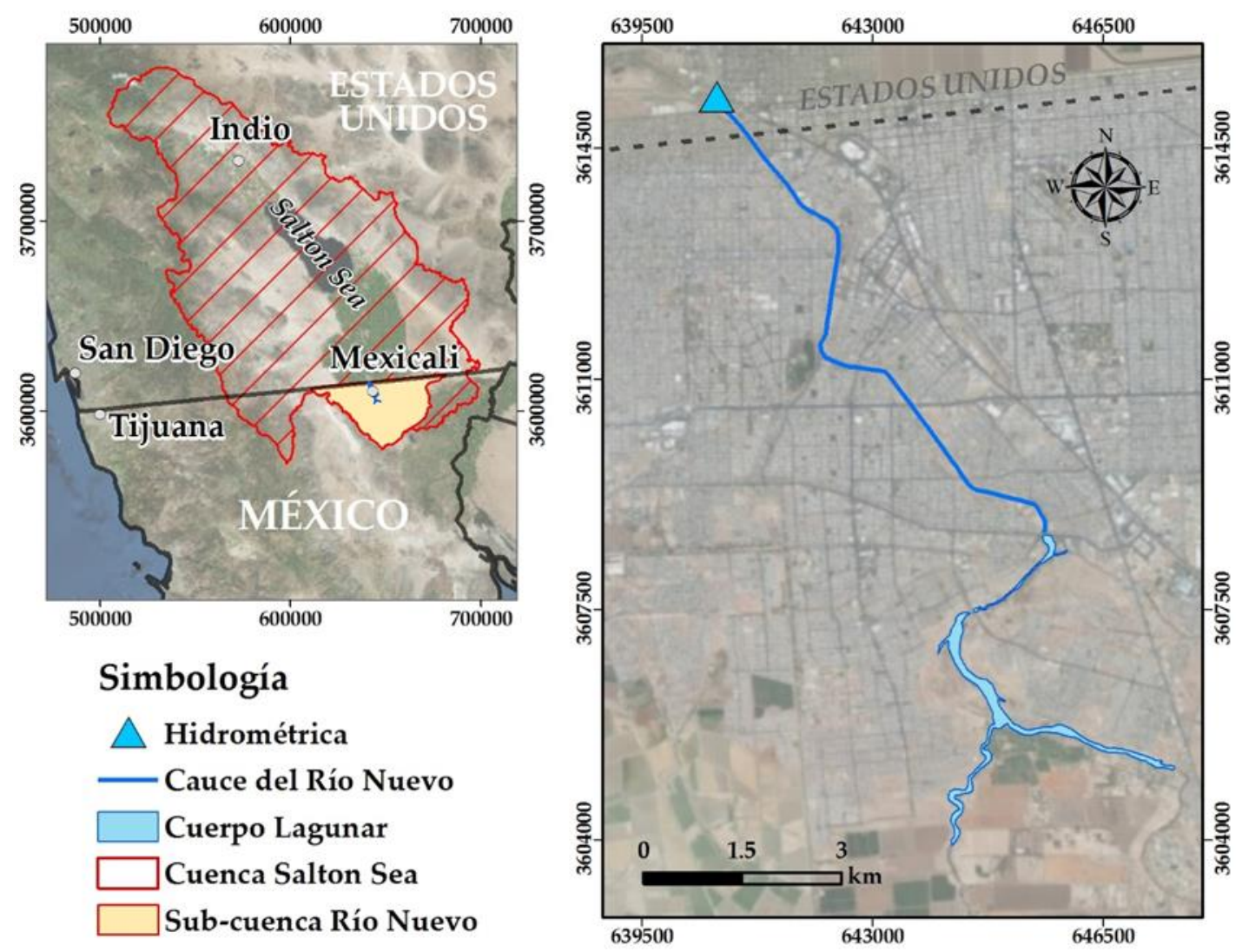

Figura 1. Localización del área de estudio.

Cabe mencionar que este río pertenece al delta del río Colorado, que actualmente abastece a más de 30 millones de personas en Estados Unidos y México (Rodríguez-Burgueño, Shanafield, \& Ramírez-Hernández, 2017). El río Colorado suministra agua a los estados de Arizona, California, Colorado, Nuevo México, Nevada, Utah y Wyoming, en Estados Unidos, y a los estados de Baja California y Sonora, en México, suministrando agua para regar casi $22257 \mathrm{~km}^{2}$ de campos agrícolas y generando más de 4200 megawatts de generación eléctrica (U.S Department of the Interior Bureau of Reclamation, 2012). Según los datos del Instituto Nacional de Estadística e Informática (INEGI), referidos en el Censo de Población y Vivienda del año 2010, la población total en Mexicali era de 936826 habitantes, representando $29.7 \%$ de la población total del estado de Baja California.

La superficie de la subcuenca del Río Nuevo, que trasvasa en la frontera de ambos países, es de $2066.69 \mathrm{~km}^{2}$ y cuenta con una pendiente media de $9.61 \%$. La longitud de su cauce principal es de $47270 \mathrm{~m}$ y su pendiente es de $3.063 \%$ (INEGI, 2017). Cabe mencionar que este estudio considera la sección del Río Nuevo con una longitud de 14.17 km, ubicada 
geográficamente en la coordenadas UTM-WGS84-Z11N a los 3608 $660.30 \mathrm{~m}$ norte y $645613.80 \mathrm{~m}$ oeste.

\section{Materiales y métodos}

Se desarrolló un modelo hidrológico para obtener un gasto de diseño estimado en diferentes periodos de retorno, bajo el esquema del análisis regional de frecuencia, con el enfoque de los $L$-momentos. Los resultados alimentaron el modelado hidrológico e hidráulico de la cuenca del Río Nuevo para identificar las zonas de inundación sobre el cauce principal de la cuenca. La preparación e interpretación de los datos del modelo para el procesamiento y la visualización de datos geoespaciales se facilitó con el uso de un sistema de información geográfico (SIG) (Heimhuber, 2013). Se utilizaron las extensiones HEC-GeoHMS y HEC-GeoRAS para la preparación de los archivos de entrada del modelo, incluyendo los registros históricos de precipitación de 35 estaciones meteorológicas y los registros de caudal de una estación hidrométrica ubicada en el trasvase del Río Nuevo, en la frontera con Estados Unidos. En el caso de México, los registros históricos de precipitación se obtuvieron a través del Extractor Rápido de Información climatológica (ERIC III) del Instituto Mexicano de Tecnología del Agua (IMTA) y la Comisión Nacional del Agua (Conagua). En el caso de los registros de Estados Unidos, se utilizaron los datos obtenidos durante el periodo correspondiente a los años de 1930 a 2016, del archivo de datos climáticos de California (CalClim, por sus siglas en inglés). En la Figura 2 se muestra de manera esquemática la metodología implementada para este estudio. 


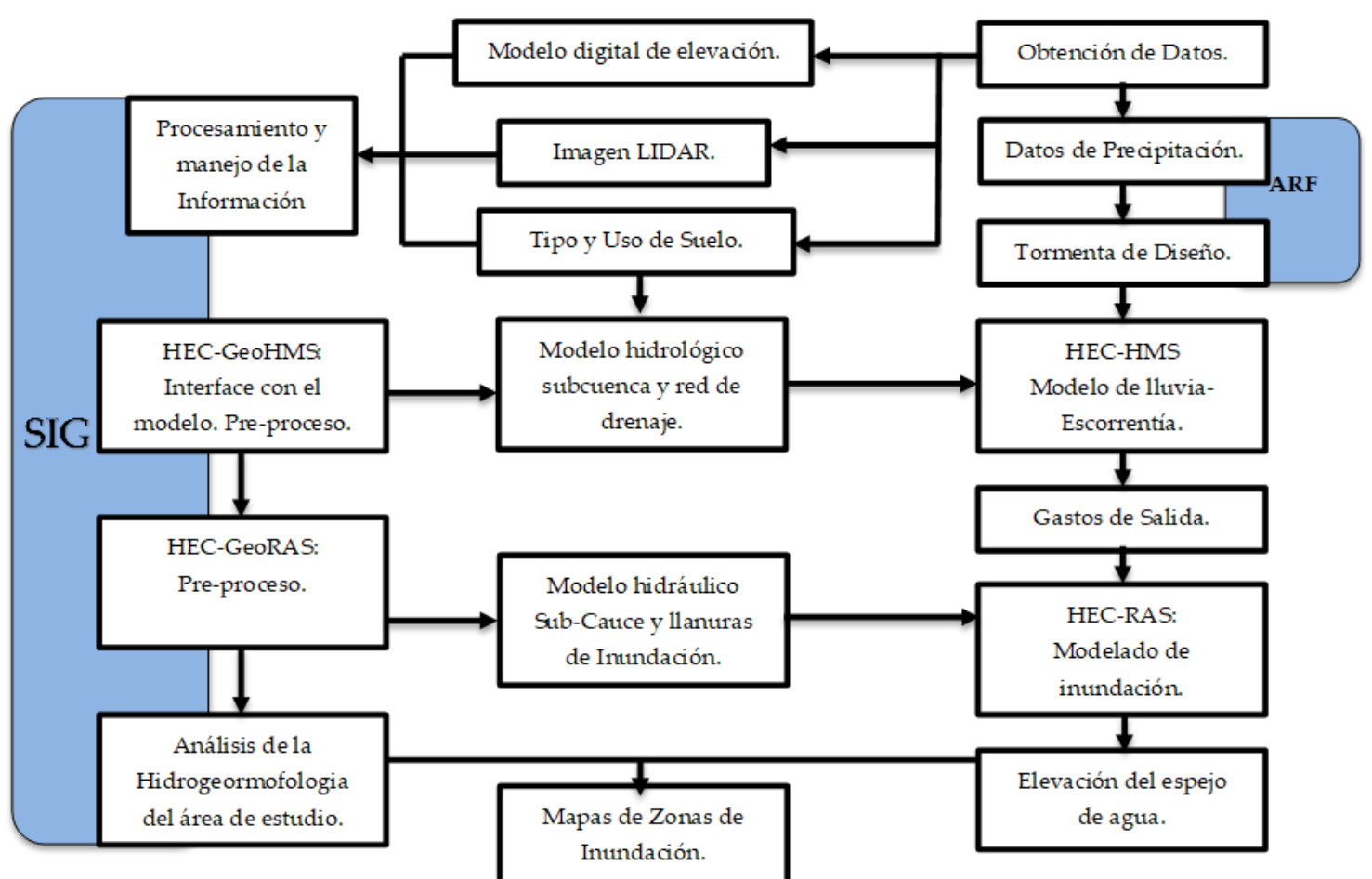

Figura 2. Diagrama del modelo hidrológico e hidráulico basado en SIG, con tormenta de diseño obtenida a través del análisis regional de frecuencia (modificado a partir de Heimhuber, 2013).

\section{Análisis regional de frecuencia con el enfoque de los L- momentos}

La mayoría de los procedimientos de análisis regional de frecuencia intenta ajustar a los datos una distribución, cuya forma se especifica a partir de un número finito de parámetros indeterminados del momento de la muestra. En particular, la asimetría (skewness) y la curtosis (kurtosis) se utilizan de modo frecuente para juzgar la cercanía de una muestra observada a una distribución postulada. Sin embargo, las estadísticas son insatisfactorias debido a que están algebraicamente delimitadas, con límites que dependen del tamaño de la muestra; en muestras pequeñas o moderadas, es inusual que asimetría y curtosis de la muestra tomen un valor cercano a los valores poblacionales (Hosking \& Wallis, 1997).

Los L-momentos o momentos lineales son un sistema alterno a los métodos tradicionales de los momentos convencionales, que caracterizan 
las formas de las distribuciones de probabilidad y determinan los parámetros de distribución. Según Hosking y Wallis (1997), los Lmomentos se consideran combinaciones lineales de los momentos ponderados. Si bien los $L$-momentos son un desarrollo relativamente reciente dentro de las estadísticas, forman la base de una teoría matemática y pueden utilizarse para facilitar el proceso de estimación en el análisis regional de frecuencia.

La metodología del ARF utilizada en el presente estudio, con enfoque en los L-momentos, se basa en los trabajos de Wallis et al. (2007), y Schaefer, Barker, Taylor y Wallis (2006), referida por Núñez-Cobo Verbist, Ramírez-Hernández, \& Hallack-Alegría (2010), y consta de cuatro etapas: 1) selección de los datos; 2) identificación de regiones homogéneas; 3 ) selección de la distribución de frecuencia, y 4) estimación de la función de cuantiles.

\section{Selección de los datos}

El análisis comienza con la categorización de los sitios en arreglos de posibles regiones homogéneas utilizando el criterio de discordancia. Dado que el número de estaciones en las regiones puede variar y la serie de datos es relativamente pequeña, se consideró analizar los valores críticos de discordancia recomendados por Hosking y Wallis (1997), según la expresión: $D_{i=\frac{1}{3}} N\left(U_{i}-\bar{U}\right)^{T} A^{-1}\left(U_{i}-\bar{U}\right)$, siendo el sitio $i$ discordante, si $D_{i}$ es mayor, dependiendo del número de sitios analizados en el grupo como se muestra en la Tabla 1. La medida de discordancia $\left(D_{i}\right)$ es una medida estadística basada en la diferencia entre los índices $L$-momento de un sitio y el promedio L-momento de un grupo de sitios similares (Malekinezhad \& Zare-Garizi, 2014). Cabe mencionar que la medida de discordancia es un indicador secundario que apoya en la toma de decisión, al considerar mover una estación de una región a otra (Núñez-Cobo, Verbist, RamírezHernández, \& Hallack-Alegría, 2010).

Tabla 1. Valores críticos de discordancia $\left(D_{i}\right)$ según Hosking y Wallis (1997).

\begin{tabular}{|c|c|c|c|}
\hline $\begin{array}{c}\text { Número de } \\
\text { sitios }\end{array}$ & $\begin{array}{c}\text { Valor } \\
\text { crítico }\end{array}$ & $\begin{array}{c}\text { Número de } \\
\text { sitios }\end{array}$ & $\begin{array}{c}\text { Valor } \\
\text { crítico }\end{array}$ \\
\hline 5 & 1.333 & 11 & 2.632 \\
\hline 6 & 1.648 & 12 & 2.757 \\
\hline
\end{tabular}




\begin{tabular}{|c|c|c|c|}
\hline 7 & 1.917 & 13 & 2.869 \\
\hline 8 & 2.140 & 14 & 2.971 \\
\hline 9 & 2.329 & 15 & 3 \\
\hline 10 & 2.491 & & \\
\hline
\end{tabular}

\section{Identificación de regiones homogéneas}

Se utilizó el criterio de estacionalidad y magnitud de la precipitación media anual para el agrupamiento de estaciones. Las medidas de heterogeneidad utilizadas han sido desarrolladas como indicadores del grado de heterogeneidad en las relaciones de los $L$-momentos para un grupo de mediciones (Schaefer, Barker, Taylor, \& Wallis, 2006). El criterio de homogeneidad en este estudio se basa en el trabajo de Wallis et al. (2007), que sugiere los rangos presentados en la Tabla 2.

Tabla 2. Valores críticos de heterogeneidad actualizados (modificado de Núñez-Cobo et al., 2010).

\begin{tabular}{|c|c|}
\hline Heterogeneidad & Wallis et al., 2007 \\
\hline Homogénea & $H<2$ \\
\hline $\begin{array}{c}\text { Posiblemente } \\
\text { heterogénea }\end{array}$ & $2<H<3$ \\
\hline Heterogénea & $H>3$ \\
\hline
\end{tabular}

Las estadísticas $H 1$ y $H 2$ miden la variabilidad relativa del coeficiente de $L$-variación ( $L-C v$, en inglés) y $L$-Skewness (equivalente al coeficiente de asimetría convencional) observadas (Núñez-Cobo et al., 2010) para sitios en una subregión, calculada según la siguiente expresión propuesta (Hosking \& Wallis, 1997): $V=\left\{\sum_{i=1}^{N} n_{i}\left(\tau^{(i)}-\tau^{R}\right)^{2} / \sum_{i=1}^{N} n_{i}\right\}^{1 / 2}$, donde se calcula la desviación estándar de la muestra ponderada en el sitio, siendo $N$ el número de sitios propuestos en la región; $i$ representa el sitio que cuenta con un registro $n_{i}$ y muestras de los $L$-momentos-ratios $\tau^{(i)}, \tau_{3}^{i}, \tau_{4}^{i}$. El promedio regional de $L$-asimetría y $L$-curtosis, ponderado a la longitud del registro.

Estas medidas compararon la variabilidad observada con la esperada de una muestra mayor tomada de una región homogénea, representada por la distribución Kappa, según la propuesta de Hosking y Wallis de 1997 (Schaefer et al., 2006). Para este estudio, uno de los criterios utilizados 
en la integración de regiones homogéneas fue que las estaciones que la integran no presenten propiedades para hacerlas discordantes con las demás que la conforman.

\section{Selección de la distribución de frecuencia}

Después de la identificación de las regiones homogéneas, se identificó la distribución de probabilidad apropiada para describir el comportamiento de los datos climáticos de interés. La distribución de frecuencia se eligió basándose en el diagrama de los $L$-momentos-ratio y la medida de bondad de ajuste $Z^{\text {Dist }}$. La bondad de ajuste juzga cuán bien la $L$-curtosis teórica de una distribución coincide con la $L$-curtosis promedio regional de los datos observados (Malekinezhad \& Zare-Garizi, 2014). La bondad de ajuste se obtuvo según la expresión: $Z^{D I S T}=\left(\tau_{4}^{D I S T}-t_{4}^{R}+B_{4}\right) / \sigma_{4}$, donde utilizó el estadístico de la prueba de bondad de ajuste $Z$, que consistió en indicar una distribución como "aceptable" para $Z \leq 1.64$, la cual corresponde a la falla de rechazo de la distribución hipotética, con un nivel de significancia de 90\% (Hosking \& Wallis, 1997). En este estudio se utilizaron cuatro distribuciones de probabilidad que mejor se ajustaron a los datos (Tabla 3), al utilizar la prueba de bondad de ajuste antes mencionada.

Tabla 3. Distribuciones de probabilidad.

\begin{tabular}{|c|c|c|}
\hline $\begin{array}{c}\text { Normal } \\
\text { generalizada }\end{array}$ & $f(x)=\frac{1}{\sigma \sqrt{2 \pi}} \exp \left(-\frac{(x-\mu)^{2}}{2 \sigma^{2}}\right)$ & $-\infty \leq x \leq \infty$ \\
\hline Pearson tipo III & $f(x)=\frac{\lambda^{\beta}(x-\varepsilon)^{\beta-1} e^{-\lambda(x-\varepsilon)}}{\Gamma(\beta)}$ & $x \geq \epsilon$ \\
\hline $\begin{array}{c}\text { Generalizada } \\
\text { de valor extremo }\end{array}$ & $\alpha^{-1} e^{-(1-k) y-e^{-y}}$ & $-\infty<x \leq \xi+\alpha / k k>0$ \\
& $y=\left\{\begin{array}{r}-k^{-1} \log \{1-k(x-\xi) / \alpha\} \\
(x-\xi) / \alpha \quad k=0\end{array}\right.$ & $-\infty<x<\infty k=0$ \\
& $k+\alpha / k \leq x<\infty k<0$ \\
\hline
\end{tabular}




\begin{tabular}{|c|c|c|}
\hline & & Límite superior es: \\
& $\xi+\alpha / k$ sik $>0, \infty$ si $\mathrm{k} \leq 0 ;$ \\
Kappa de cuatro & $f(x)=\alpha^{-1}\{1-k(x$ & \\
parámetros & $-\xi) / \alpha\}^{1 / k-1}\{F(x)\}^{1-h}$ & Límite inferior es: \\
& $\xi+\alpha\left(1-h^{-k}\right) / k$ si $\mathrm{h}<0$ \\
& $\xi+\alpha / k$ si $\mathrm{h} \leq 0$ y $\mathrm{k}<0$, \\
& & $y-\infty$ si $\mathrm{h} \leq 0$ y $\mathrm{k} \geq 0$ \\
\hline
\end{tabular}

\section{Estimación de la función de cuantiles}

Una vez identificada la distribución de probabilidad para la región homogénea, se determinaron los cuantiles para diferentes periodos de retorno usando el método Índice de Avenidas, el cual se expresa como $Q_{i}(F)=\breve{\mu}_{i} g(F)$, donde $Q_{i}(F)$ es el cuantil regional estimado; $\breve{\mu}_{i}$ es la media de la distribución de frecuencia del sitio, y $q(F)$ es la curva de crecimiento de la región (Núñez-Cobo et al., 2010).

\section{Modelo hidrológico}

Las simulaciones hidrológicas se realizaron empleando la herramienta HEC-HMS 3.2 del Hydrologic Engineering Center, representando el proceso de lluvia escorrentía de manera simplificada, simulando el hidrograma que se produce en un determinado punto de la red fluvial, como consecuencia de un episodio de precipitación (López et al., 2012). El modelo en HEC-HMS consideró cuatro componentes: 1) modelo de la cuenca; 2) modelo meteorológico, que indicó la precipitación de entrada requerida por un elemento de la subcuenca; 3 ) especificación de control con el cual se determinó el inicio y periodo de simulación, y 4) datos de entrada utilizados para proporcionar las variables hidrológicas, como datos de series de tiempo (USACE, 2016).

\section{Determinación de los parámetros de tipo de uso de suelo}


La modelación del escurrimiento se basó en el método del Número de Curva (CN) del Servicio de Conservación de Suelos (SCS). Este método se basa en la ecuación de balance de agua, la cual dentro del HEC-HMS estima la precipitación en exceso o efectiva en función de la precipitación acumulada, cobertura del suelo, uso del suelo, y humedad antecedente (Magaña-Hernández, Bá, \& Guerra-Cobián, 2013). A fin de determinar con mayor precisión el CN, se obtuvieron los tipos de suelo, el tamaño de sus áreas y su porcentaje de superficie en la zona. Para ello se procesaron 1500 polígonos con un SIG, sobreponiendo la capa de uso de suelo del Instituto Nacional de Geografía Estadística e Informática (INEGI) a las imágenes obtenidas a través de la plataforma Google Earth. Después, para la definición del $\mathrm{CN}$, se consideraron los valores de la tabla de regiones semiáridas del Natural Resources Conservation Service (NRCS, 1986). Finalmente, se realizó una calibración del modelo hidrológico ajustando el CN, ya que esta variable es el parámetro más sensible (López et al., 2012).

En las especificaciones de control del HEC-HMS se definió el lapso y la fecha de la ejecución de la simulación, siendo el modelo meteorológico la representación de la precipitación. El modelo de cuenca se preparó con la extensión HEC-GeoHMS utilizando la herramienta de fusión de microcuencas, siguiendo las restricciones de adyacencia y confluencia para facilitar el manejo de la información espacial. Los resultados del modelo comprendieron hidrogramas de escorrentía para cada subcuenca, así como representaciones gráficas y numéricas de precipitaciones, pérdidas y escurrimiento directo. Por su parte, el tiempo de concentración se calculó con el método de Kirpich, donde $T c=0.000323\left(\frac{L^{0.77}}{S^{0.385}}\right)$, obteniendo el tiempo de retardo expresado como $0.6 * T c$.

\section{Delimitación de subcuenca y microcuencas}

Una cuenca hidrográfica suele ser delineada como varias subcuencas antes de aplicar el análisis con el HEC-HMS (Patel, 2009). Por lo tanto, la delimitación de las cuencas y redes de drenaje fueron obtenidas utilizando un MDE generado a partir de datos de elevación de alta resolución (LiDAR) de 5 × 5 metros (INEGI, 2010) mediante un SIG.

\section{Modelo hidráulico}


Los modelos hidráulicos se utilizaron para simular el comportamiento del flujo en el canal principal y la llanura de inundación del Río Nuevo (Alaghmand, Abdullah, Abustan, \& Vosoogh, 2010). Los gastos de diseño de escorrentía generados, se utilizaron como entrada para el modelado en HEC-RAS. La geometría del río fue procesada usando la extensión del HEC-GeoRAS. La realización del modelo hidráulico se constituyó por tres procedimientos fundamentales, como se presenta a continuación.

\section{Preproceso}

Se utilizaron los datos geométricos del modelo hidráulico, conformados por el eje del río, secciones transversales, distancia de separación entre secciones, puntos de control, así como el coeficiente de Manning y coeficientes de contracción/expansión.

\section{Proceso-simulación hidráulica con HEC-RAS}

Durante la etapa del proceso se establecieron las condiciones de frontera aguas arriba y aguas abajo en la modalidad de flujo permanente. Cabe mencionar que debido a la falta de referencia del nivel del agua se optó por la condición de frontera de tirante crítico (USACE, 2016). Posteriormente se definió el régimen de flujo mixto, a fin de correr la modelación y como parte de este apartado, finalmente se llevó a cabo la calibración del modelo mediante la relación caudal-tirante de agua. El caudal se obtuvo por medio de mediciones in situ, utilizando un velocímetro Doppler acústico y los resultados fueron utilizados para ajustar el modelo matemático de tal manera que reproduzca el fenómeno en estudio (Parhi, Sankhua, \& Roy, 2012).

\section{Posproceso}


Una vez calibrado el modelo hidráulico se elaboraron mapas de inundación (profundidad) para los diferentes escenarios de flujo. El modelado hidráulico en esta investigación se realizó sin considerar la infraestructura del embovedado del Río Nuevo debido a que en regiones áridas y semiáridas los eventos de inundaciones repentinas son causados por tormentas de alta intensidad y corta duración con alto grado de variabilidad espacial. Estas tormentas suelen presentar crecidas intensas en tiempos muy cortos incluso para cuencas grandes, lo que resulta en cantidades significativas de sedimentos transportados, afectando la infraestructura hidráulica de las instalaciones aguas abajo (Maksimovic, 2001). La modelación hidráulica —con una extensión de 14.17 km del cauce principal del Río Nuevo, incluyendo el cuerpo lagunar al sur de la ciudad-se analizó mediante 143 secciones transversales distribuidas en intervalos aproximados a los 100 metros.

\section{Resultados y discusión}

El estado del arte indica que la generación espacial de la escorrentía en las zonas áridas y semiáridas no es uniforme. Sin embargo, los mecanismos de precipitación dominantes son usualmente tormentas de alta intensidad y corta duración (Wang, Zhang, \& Baddo, 2016), condiciones que se cumplen en el área de estudio. Cabe destacar que este trabajo es el primero con tales características que se realiza en la zona, acoplando el modelo hidrológico con el hidráulico, por lo que se espera que sus resultados sean usados como referencia para análisis posteriores.

\section{Análisis regional de frecuencias}

Debido a la limitada infraestructura en estaciones meteorológicas y disponibilidad de registros de precipitación en México, el enfoque probabilístico del análisis regional de frecuencia se contempla como una opción válida para estimar la ocurrencia de un evento de precipitación extremo, que será empleado como dato de entrada de un modelo 
hidrológico. Según los resultados del ARF, se definieron 20 estaciones en la cuenca del Salton Sea consideradas homogéneas (Tabla 4). Seleccionándose la distribución Pearson Tipo III, que fue validada con las funciones Normal Generalizada y Generalizada de Valor Extremo (The Salton Sea Authority, 2015).

Tabla 4. Estaciones de la región homogénea utilizada en el estudio.

\begin{tabular}{|c|c|c|c|c|c|c|c|}
\hline Estación & $\begin{array}{c}\text { Latitud } \\
\text { norte }\end{array}$ & $\begin{array}{l}\text { Longitud } \\
\text { oeste }\end{array}$ & PMA & L-CV & L-skew & L-kurt & $\begin{array}{c}\text { Discordancia } \\
\text { (Di) }\end{array}$ \\
\hline Brawley & 634781.34 & 3647110.12 & 47.1 & 0.366 & 0.242 & 0.175 & 0.26 \\
\hline Bataquez & 681308.65 & 3603156.81 & 36.7 & 0.413 & 0.227 & 0.144 & 1.48 \\
\hline Calexico & 643996.85 & 3617742.02 & 44.7 & 0.357 & 0.171 & 0.139 & 0.33 \\
\hline Col. Rodríguez & 684677.21 & 3588577.52 & 36.1 & 0.402 & 0.183 & 0.174 & 1.75 \\
\hline Deep Canyon Lab & 557860.08 & 3723635.24 & 84.4 & 0.315 & 0.278 & 0.224 & 1.82 \\
\hline Desert Resort RG & 578003.43 & 3721116.77 & 50.8 & 0.334 & 0.212 & 0.155 & 0.19 \\
\hline Ejido Islitas & 696293.9 & 3581476.88 & 46.0 & 0.361 & 0.234 & 0.236 & 1.14 \\
\hline El Centro 2 & 634674.78 & 3627480.63 & 45.3 & 0.323 & 0.112 & 0.083 & 2.00 \\
\hline Hayfield Pump PL & 627051.03 & 3730180.46 & 65.1 & 0.345 & 0.211 & 0.135 & 0.32 \\
\hline Imperial Airport & 633815.99 & 3635564.07 & 43.1 & 0.317 & 0.144 & 0.122 & 1.00 \\
\hline Indio Fire Station & 538732.66 & 3729969.65 & 50.7 & 0.382 & 0.237 & 0.103 & 1.93 \\
\hline Mecca 2 & 585664.6 & 3714972.54 & 47.8 & 0.386 & 0.175 & 0.148 & 0.90 \\
\hline Mxli (Río Nuevo) & 644499.45 & 3615420.52 & 49.2 & 0.313 & 0.208 & 0.174 & 0.53 \\
\hline Mxli C. Agrícola & 617926.28 & 3602097.2 & 68.7 & 0.354 & 0.239 & 0.214 & 0.48 \\
\hline Niland & 637453.84 & 3683078.33 & 44.5 & 0.358 & 0.265 & 0.222 & 0.79 \\
\hline Ocotillo 2 & 593590.88 & 3623570.42 & 66.5 & 0.333 & 0.186 & 0.194 & 0.66 \\
\hline Ocotillo Wells & 580855.45 & 3668250.7 & 43.5 & 0.310 & 0.147 & 0.123 & 1.11 \\
\hline Palm Springs & 545342.18 & 3743082.29 & 81.2 & 0.343 & 0.253 & 0.125 & 1.85 \\
\hline Presa Morelos & 712686.4 & 3620860.17 & 33.9 & 0.387 & 0.225 & 0.161 & 0.48 \\
\hline Yuma Valley & 713970.32 & 3622219.06 & 49.2 & 0.317 & 0.212 & 0.217 & 0.99 \\
\hline
\end{tabular}

Después se determinaron los cuantiles correspondientes a los $\operatorname{Tr}$ de 20, 50,100 y 500 años, respectivamente, que se presentan en la Tabla 5.

Tabla 5. Cuantiles correspondientes a los periodos de retorno de 20, 50,100 y 500 años.

\begin{tabular}{|c|c|c|c|c|c|}
\hline Cuantil & $\begin{array}{c}\text { Periodos de } \\
\text { retorno }\end{array}$ & $\begin{array}{c}\text { Normal } \\
\text { Generalizada }\end{array}$ & $\begin{array}{c}\text { Generalizada de } \\
\text { Valor Extremo }\end{array}$ & Pearson Tipo III & Kappa \\
\hline 0.950 & 20.00 & 2.25 & 2.25 & $\mathbf{2 . 2 6}$ & 2.26 \\
\hline 0.980 & 50.00 & 2.79 & 2.81 & $\mathbf{2 . 7 5}$ & 2.79 \\
\hline 0.990 & 100.00 & 3.20 & 3.25 & $\mathbf{3 . 1 0}$ & 3.19 \\
\hline 0.998 & 500.00 & 4.21 & 4.36 & $\mathbf{3 . 9 1}$ & 4.16 \\
\hline
\end{tabular}




\section{Características de los modelos hidrológico e hidráulico}

Se obtuvieron 20 microcuencas definidas durante el preproceso en HECGeoHMS, las cuales cuentan con una extensión variable para un área total de $562 \mathrm{~km}^{2}$. El punto de interés fue ubicado en la salida del Río Nuevo, en el trasvase a California, Estados Unidos, como se muestran en la Figura 3.

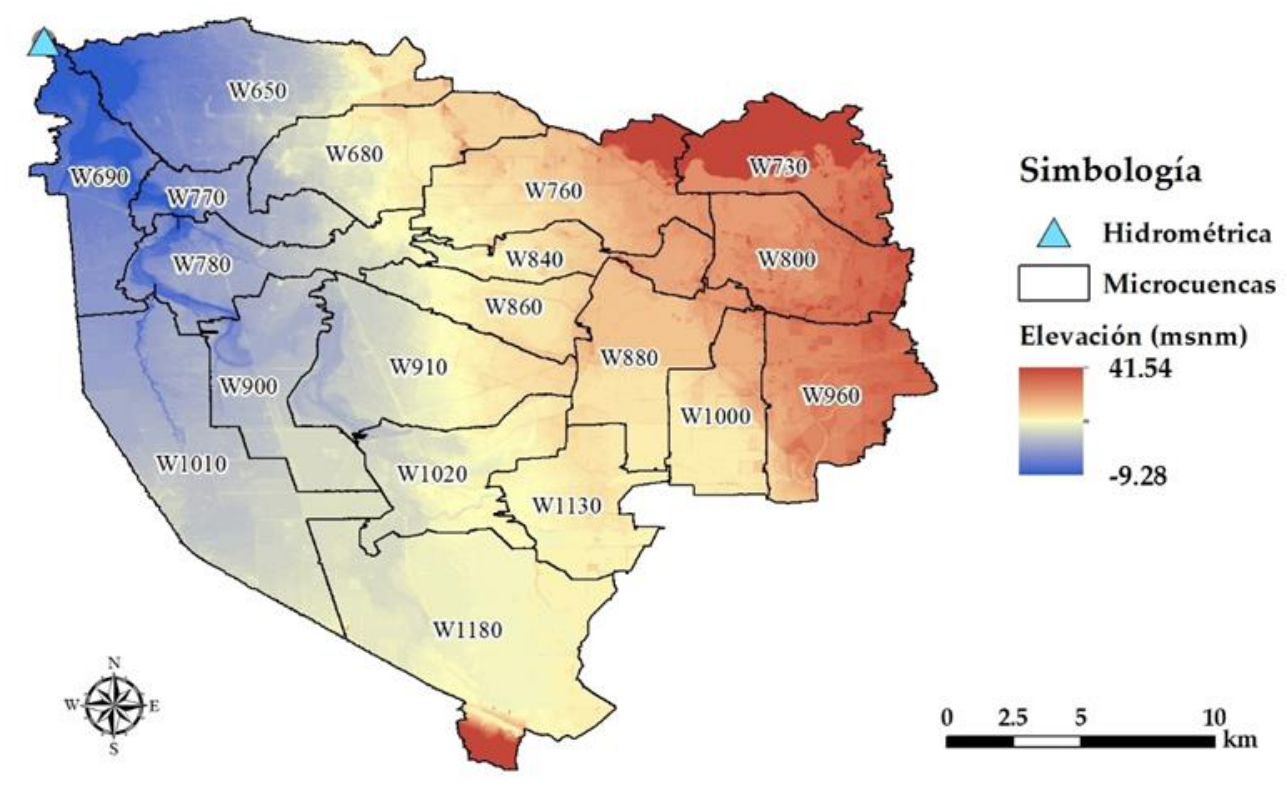

Figura 3. Microcuencas y MDE del Río Nuevo.

Una vez definidas las características físicas de la cuenca, el proyecto fue exportado al HEC-HMS, como se muestra en la Figura 4. 


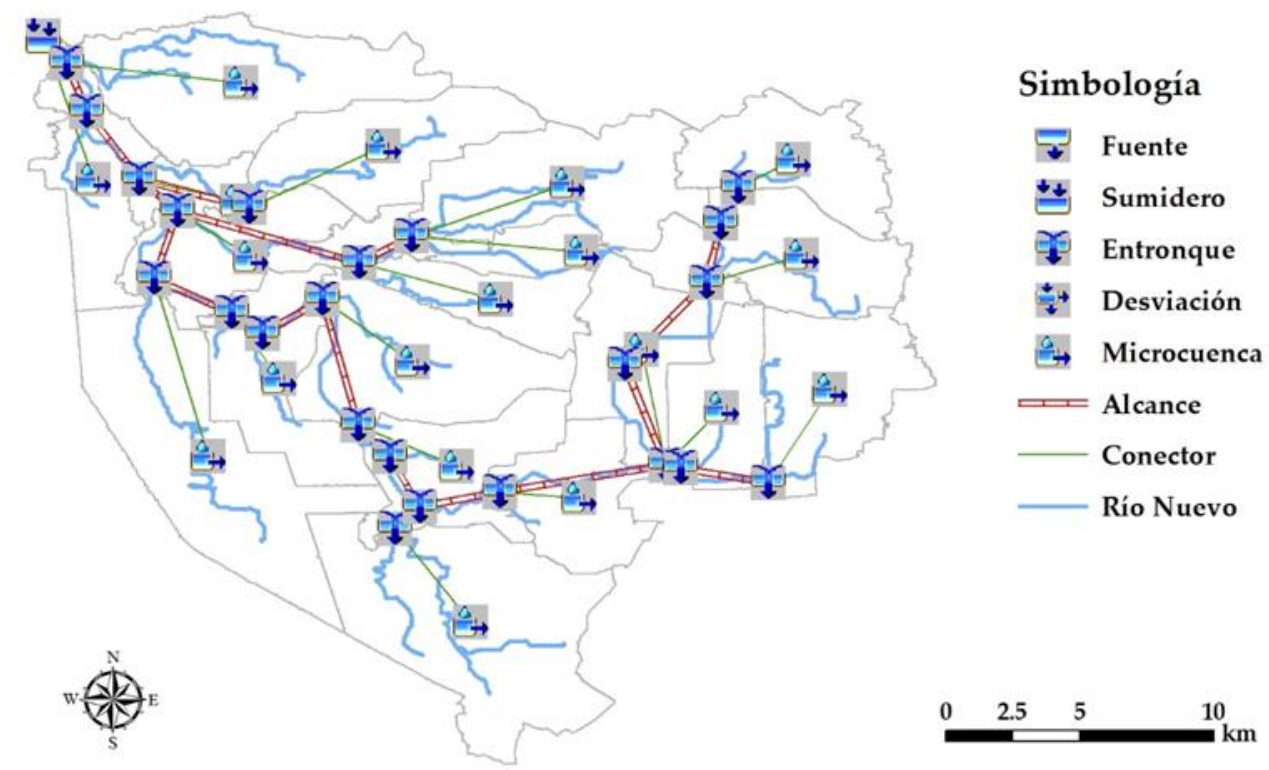

Figura 4. Modelo del HEC-HMS de la subcuenca del Río Nuevo.

Posteriormente se definieron los datos de entrada de los métodos de pérdida, transformación y enrutamiento, a fin de establecer los parámetros del modelo donde después se analizaron los diferentes periodos de retorno. Por su parte, los gastos máximos en las simulaciones sirvieron de base para el análisis hidráulico (Tabla 6). Cabe señalar que para aumentar la precisión del $\mathrm{CN}$ se propuso la abstracción mediante un SIG de los diferentes tipos de uso de suelo, a fin de disminuir los errores por la generalización de los datos propuestos en los manuales.

Tabla 6. Datos de entrada del modelo: número de curva $(C N)$; tiempo de concentración (TC); abstracción inicial (Ia); retención máxima $(S)$, y lag por microcuenca (entradas); además, salidas del modelo: área de drenaje $\left(\mathrm{km}^{2}\right)$ y gastos máximos $\left(\mathrm{m}^{3} / \mathrm{s}\right)$ por periodo de retorno analizado.

\begin{tabular}{|c|c|c|c|c|c|c|c|c|c|c|}
\hline \multirow{3}{*}{ Microcuenca } & \multicolumn{5}{|c|}{ Entradas } & \multicolumn{5}{|c|}{ Salidas } \\
\hline & \multirow{2}{*}{$C N$} & \multirow{2}{*}{$\begin{array}{c}T C \\
\text { Kirpich } \\
\text { (horas) }\end{array}$} & \multirow{2}{*}{$\underset{(\min )}{\text { Lag Time }}$} & \multirow{2}{*}{$S$} & \multirow{2}{*}{ Ia } & \multirow{2}{*}{$\begin{array}{c}\text { Área de } \\
\text { drenaje } \\
\left(\mathrm{km}^{2}\right)\end{array}$} & \multicolumn{4}{|c|}{ Gasto máximo } \\
\hline & & & & & & & $\operatorname{Tr} 20$ & $\operatorname{Tr} 50$ & $\operatorname{Tr} 100$ & $\operatorname{Tr} 500$ \\
\hline W650 & 86 & 10.58 & 380.82 & 41.35 & 8.27 & 45.10 & 8.50 & 12.20 & 14.50 & 20.60 \\
\hline W680 & 82 & 12.13 & 436.81 & 55.76 & 11.15 & 29.67 & 4.30 & 6.40 & 7.80 & 11.50 \\
\hline W690 & 82 & 8.28 & 297.91 & 55.76 & 11.15 & 24.09 & 3.50 & 5.20 & 6.30 & 9.40 \\
\hline W730 & 64 & 6.22 & 223.92 & 142.88 & 28.58 & 25.12 & 0.10 & 0.40 & 0.60 & 1.50 \\
\hline W760 & 68 & 10.38 & 373.61 & 119.53 & 23.91 & 37.02 & 1.30 & 2.60 & 3.60 & 6.40 \\
\hline W770 & 88 & 10.54 & 379.48 & 34.64 & 6.93 & 14.49 & 3.10 & 4.40 & 5.10 & 7.20 \\
\hline
\end{tabular}




\begin{tabular}{|c|c|cccc|ccccc|} 
W780 & 86 & 9.19 & 330.93 & 41.35 & 8.27 & 24.89 & 4.70 & 6.70 & 8.00 & 11.40 \\
W800 & 70 & 11.41 & 410.78 & 108.86 & 21.77 & 26.32 & 0.40 & 0.90 & 1.40 & 2.70 \\
W810 & 86 & 1.36 & 49.01 & 41.35 & 8.27 & 0.29 & 0.10 & 0.10 & 0.10 & 0.10 \\
W840 & 72 & 12.53 & 451.02 & 98.78 & 19.76 & 15.79 & 0.80 & 1.40 & 1.80 & 3.10 \\
W860 & 88 & 10.26 & 369.23 & 34.64 & 6.93 & 16.67 & 3.60 & 5.00 & 5.90 & 8.20 \\
W880 & 72 & 12.31 & 443.21 & 98.78 & 19.76 & 27.47 & 0.70 & 1.40 & 2.00 & 3.60 \\
W900 & 77 & 11.69 & 420.96 & 75.87 & 15.17 & 27.40 & 2.70 & 4.40 & 5.50 & 8.50 \\
W910 & 82 & 11.84 & 426.12 & 55.76 & 11.15 & 37.76 & 5.50 & 8.20 & 9.90 & 14.70 \\
W960 & 68 & 10.80 & 388.98 & 119.53 & 23.91 & 29.76 & 0.30 & 0.80 & 1.30 & 2.60 \\
W1000 & 76 & 9.75 & 350.98 & 80.21 & 16.04 & 20.44 & 0.80 & 1.40 & 1.90 & 3.20 \\
W1010 & 78 & 19.21 & 691.70 & 71.64 & 14.33 & 54.20 & 5.90 & 9.30 & 11.50 & 17.70 \\
W1020 & 82 & 10.24 & 368.81 & 55.76 & 11.15 & 25.94 & 2.60 & 4.00 & 4.90 & 7.60 \\
W1130 & 80 & 8.68 & 312.59 & 63.50 & 12.70 & 22.22 & 1.50 & 2.40 & 3.10 & 5.00 \\
W1180 & 70 & 28.22 & 1015.91 & 108.86 & 21.77 & 60.38 & 1.70 & 3.20 & 4.40 & 7.80 \\
\hline Total
\end{tabular}

\section{Determinación de las zonas de inundación por periodo de retorno}

Los peligros de inundación en zonas áridas y semiáridas son poco entendidos debido a la falta de información acerca de su comportamiento hidrológico e hidráulico, e inciden en la vulnerabilidad de la infraestructura ubicada en las llanuras de inundación. Así, este tipo de análisis es necesario para ubicar las zonas expuestas a riesgos por escorrentía asociadas con inundaciones repentinas. En el caso del cauce del Río Nuevo, tales zonas se definieron al usar el acoplamiento del modelo hidrológico e hidráulico, como se presenta a continuación.

Las profundidades y al área de inundación para los diferentes periodos de retorno analizados se muestran en la Tabla 7 , donde se observa que durante el periodo de retorno de 500 años, el rango de profundidad alcanzó hasta los 6.0 metros y una superficie inundable de 237.83 ha; mientras que en el de 20 años, la profundidad alcanzó 4.80 metros y un área de 190.55 ha.

Tabla 7. Áreas de inundación y profundidades para cada periodo de retorno analizado.

\begin{tabular}{|c|c|c|}
\hline Periodo de retorno & Área de inundación (ha) & Rango de profundidad (m) \\
\hline 20 & 190.55 & $0.10-4.80$ \\
\hline
\end{tabular}




\begin{tabular}{|c|c|c|}
\hline 50 & 206.25 & $0.10-5.20$ \\
\hline 100 & 216.66 & $0.10-5.50$ \\
\hline 500 & 237.83 & $0.10-6.00$ \\
\hline
\end{tabular}

En las siguientes figuras se muestra la representación de la zona inundable para los periodos de retorno de 20 y 500 años, mismas que muestran las profundidades mínimas y máximas obtenidas en este estudio (Figura 5).

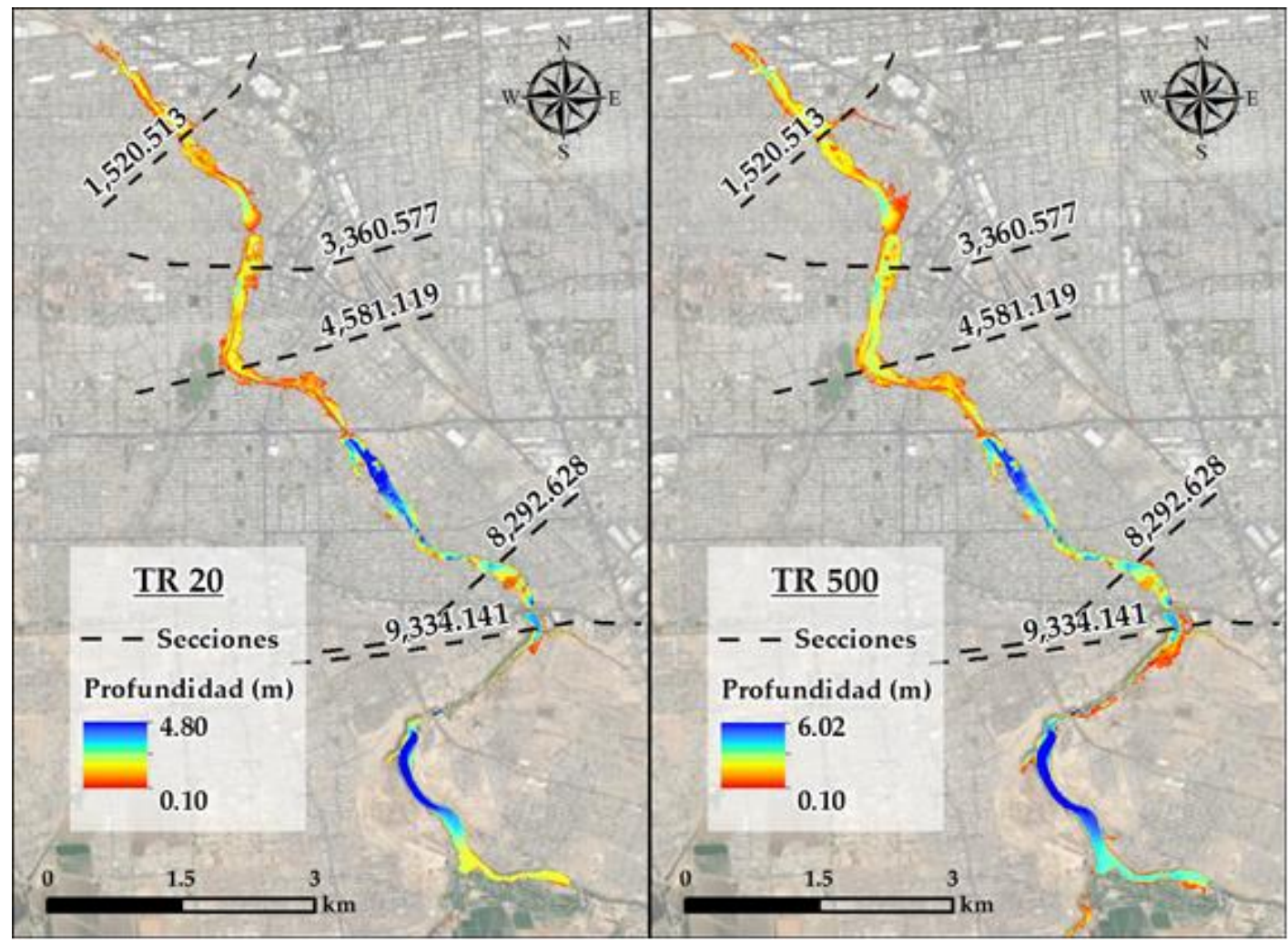

Figura 5. Extensión de la inundación y distribución de profundidad generada por HEC-RAS para eventos con periodos de retorno de 20 y 500 años.

Por su parte, en la Figura 6, Figura 7, Figura 8, Figura 9 y Figura 10 se presenta la simulación hidráulica en cinco sitios de importancia sobre el cauce del Río Nuevo por medio de las secciones transversales (RS): 1 $520.513,3360.577,4581.119,8292.628$ y 9334.141 . Cabe mencionar que a partir del periodo de retorno de 20 años hasta el de 500 se observó un comportamiento similar con variaciones en la profundidad de las zonas inundadas debido a que se identifica que el cauce está definido por pendientes pronunciadas en las márgenes este y oeste. 


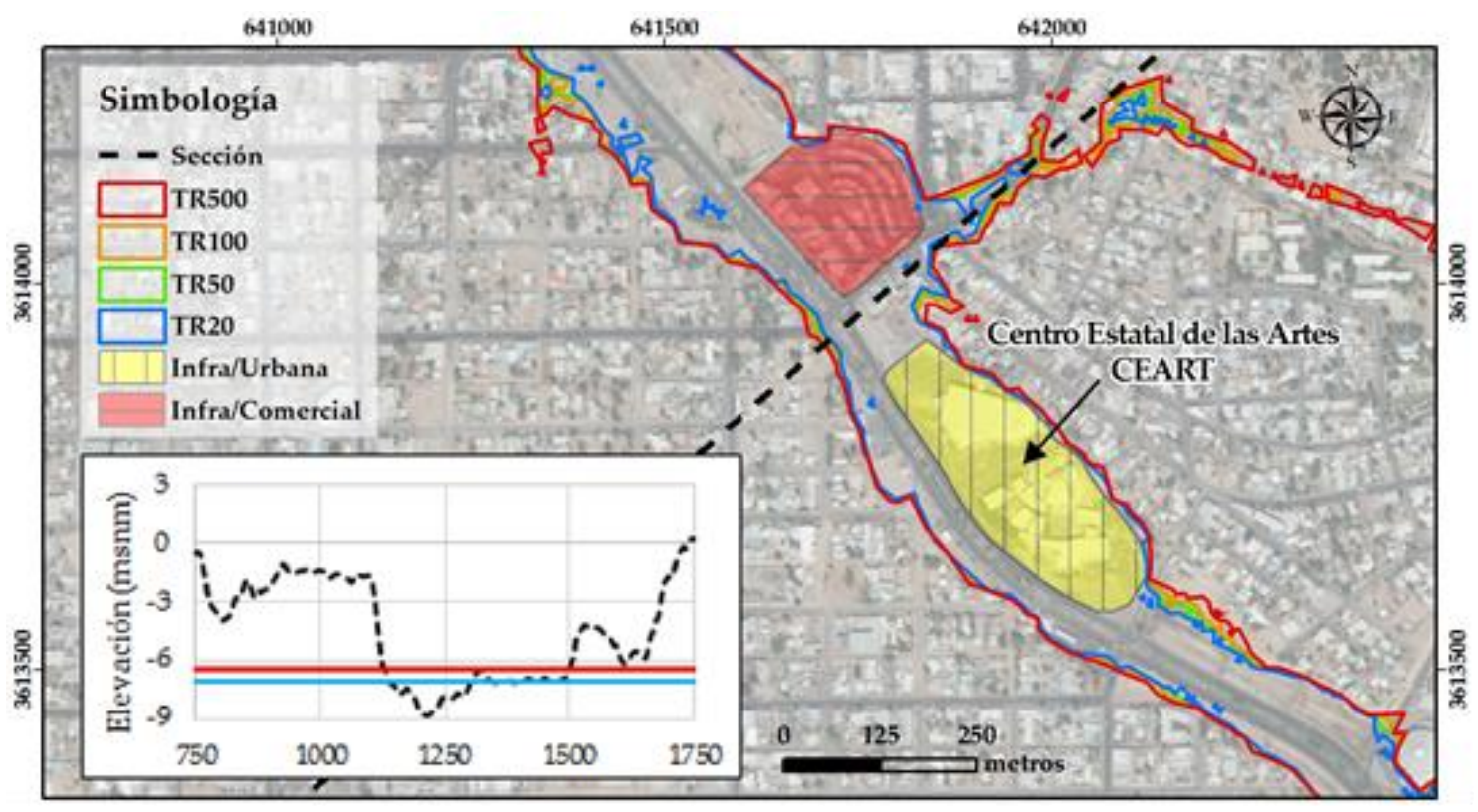

Figura 6. Zonas inundables correspondientes a la sección 1 520.513, con afectaciones a infraestructura comercial y urbana.

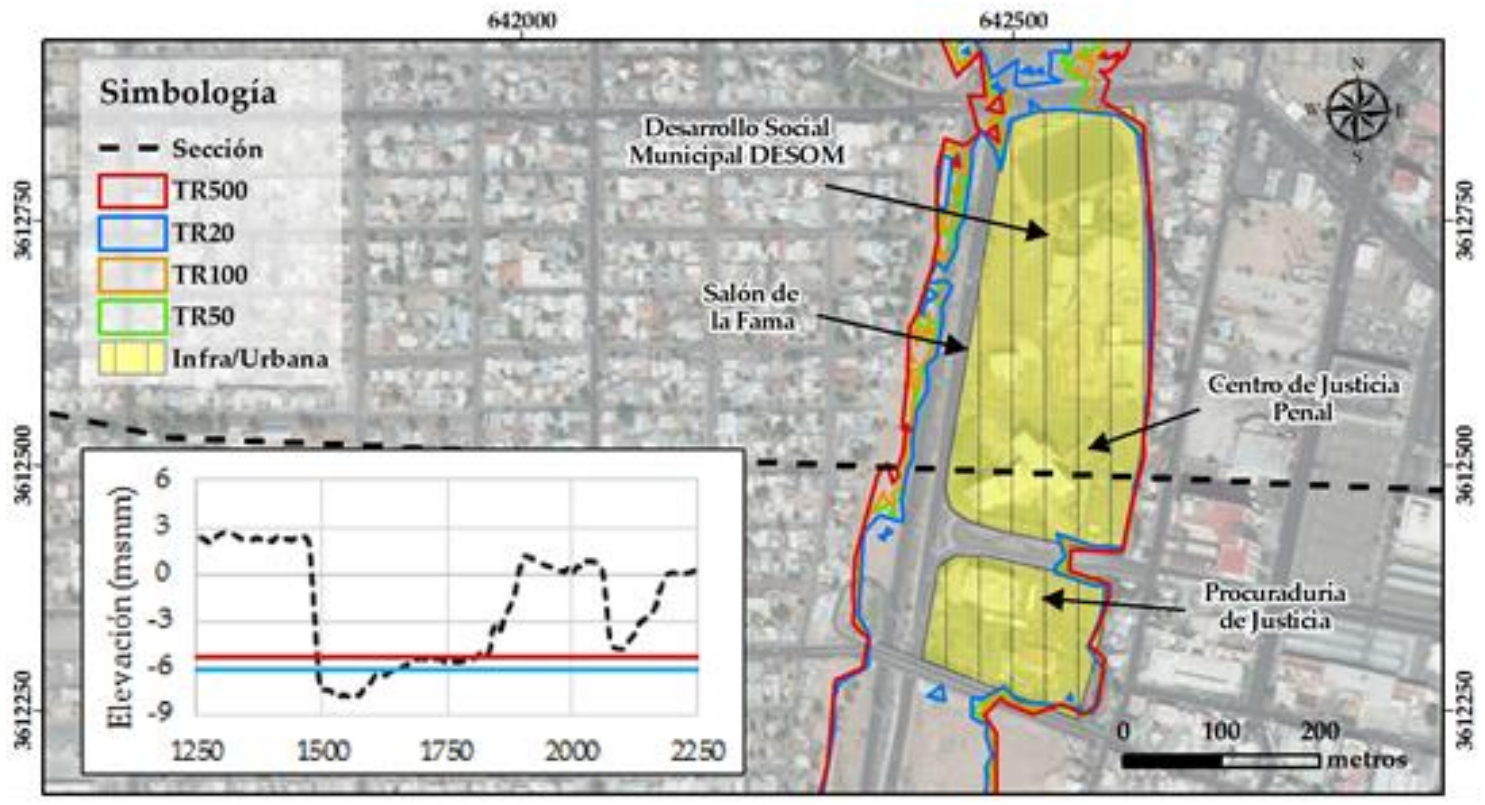

Figura 7. Zonas inundables en la sección 3 360.577, con afectaciones urbanas (edificios públicos e instalaciones deportivas). 


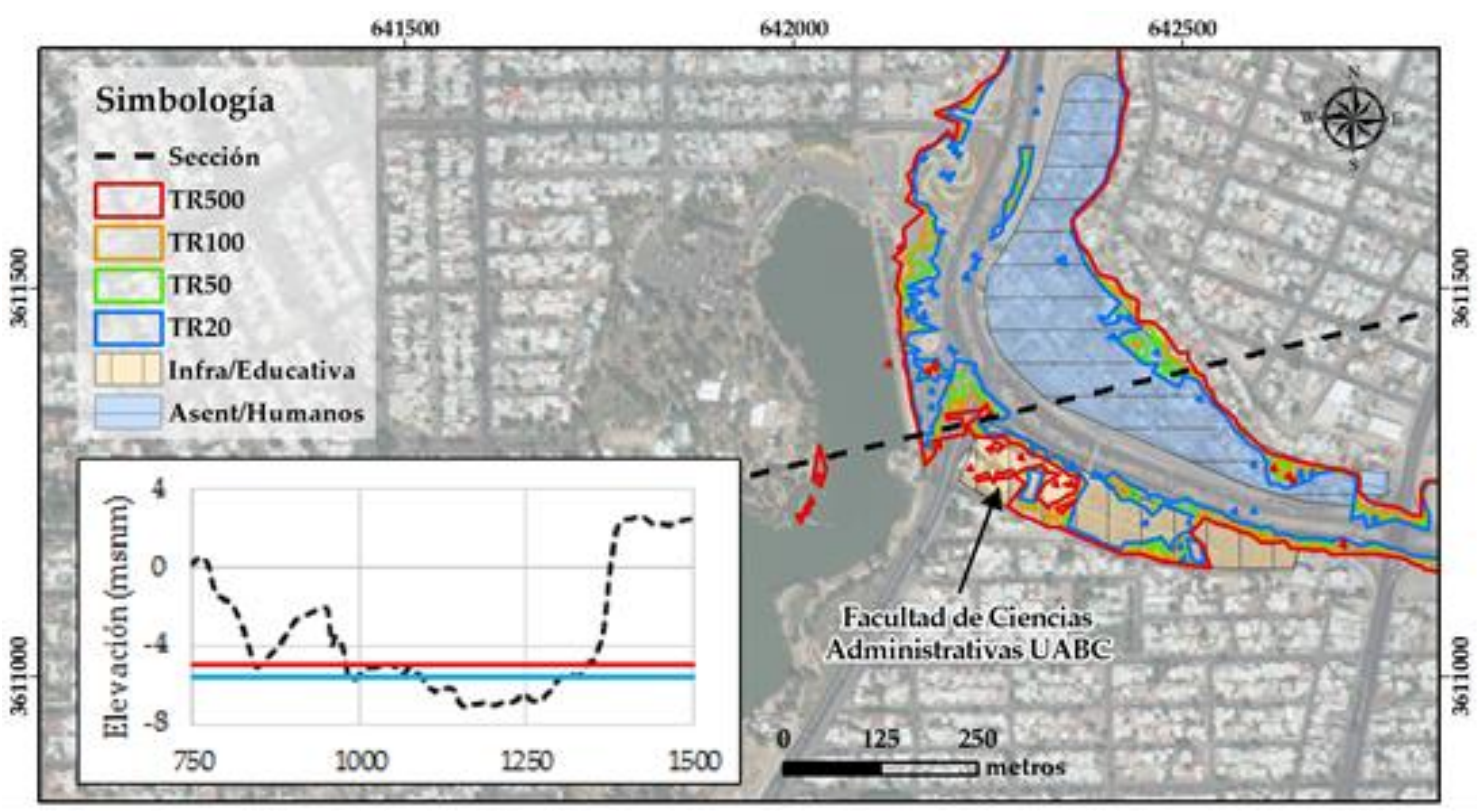

Figura 8. Zonas inundables de la sección 4 581.119, con afectaciones en asentamientos humanos e infraestructura educativa (asentamientos humanos con unas 200 familias).

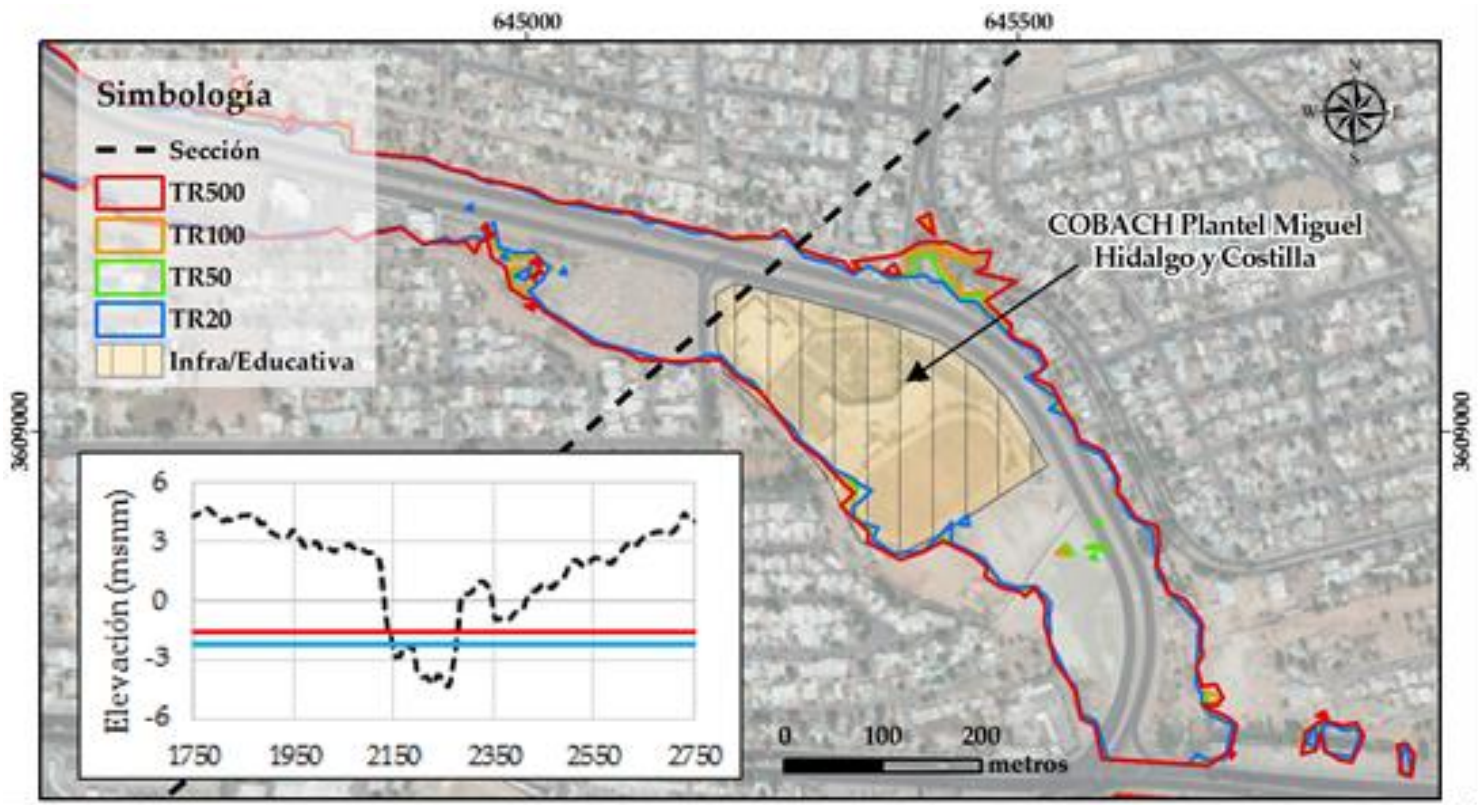

Figura 9. Zonas inundables correspondientes a la sección 8 292.628, con afectaciones en infraestructura educativa. 


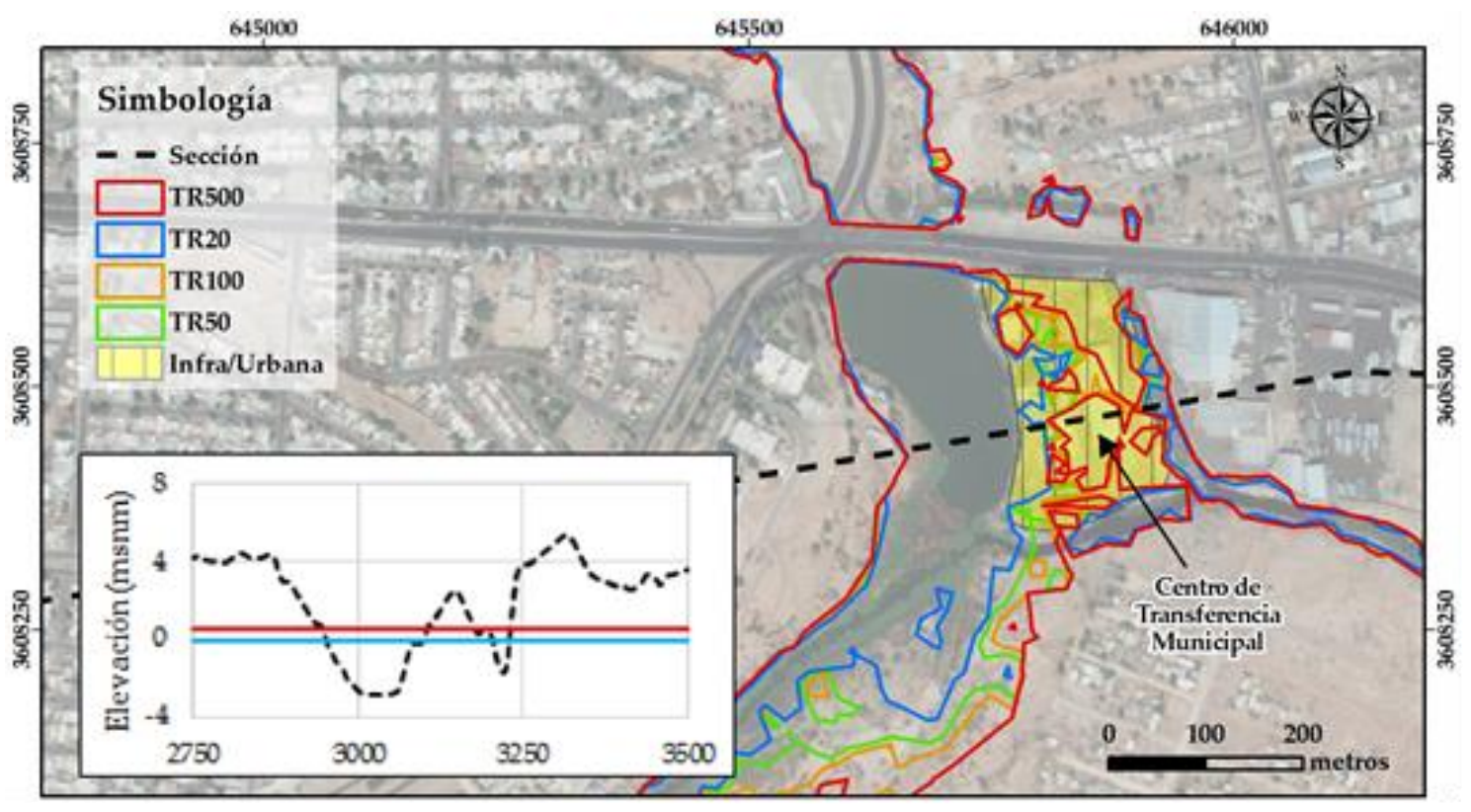

Figura 10. Zonas inundables correspondientes a la sección 9 334.141, con afectaciones en infraestructura urbana (Centro de transferencia de Residuos Sólidos Urbanos de Mexicali).

\section{Conclusiones}

El apoyo de un ARF con enfoque en los $L$-momentos permite alimentar un modelo hidrológico e hidráulico en una zona con pocas estaciones climatológicas y registros históricos de información. Además, es una herramienta útil en el procesamiento de datos y permite dar mayor certeza al identificar los cuantiles de los periodos de retorno y estimar la altura de la precipitación, misma que será utilizada como la base del modelo hidrológico. Cabe mencionar que el manejo de la información del SIG mediante las extensiones HEC-GeoRAS y HEC-GeoHMS permitió llevar a cabo la preparación de los datos de entrada para el acoplamiento del modelo hidrológico HEC-HMS con el modelo hidráulico en HEC-RAS. El resultado de este acoplamiento de modelos en la región semiárida de la cuenca transfronteriza del Río Nuevo es relevante por ser la primera vez que se realiza y, por lo tanto, se espera que sea la base para futuros análisis hidrológicos e hidráulicos de la zona.

El acelerado crecimiento de la ciudad es condicionante en el incremento de sitios impermeables de la zona urbana y del volumen de descarga de 
la escorrentía. Por lo cual, se recomienda evaluar y ajustar periódicamente el $\mathrm{CN}$ por microcuenca, según la metodología propuesta en este trabajo, con especial atención en las zonas aledañas a los escurrimientos principales dentro del núcleo urbano.

Si bien es cierto que el aumento en la magnitud de la precipitación en los periodos de retorno de 20, 50, 100 y 500 años generaron aumentos en el volumen de descarga, el área de inundación no se incrementó de modo significativo debido a que el cauce principal del Río Nuevo se encuentra definido por taludes pronunciados en sus márgenes este y oeste. Por lo tanto, la amenaza más importante identificada en un evento extremo de precipitación se relaciona con la profundidad del agua, que alcanza alturas de 4.80 m para el $T R$ de 20 años hasta 6.0 metros en un $T R$ de 500 años. Cabe mencionar que el incremento de profundidad durante estos eventos representa una amenaza, pues en el área se ha desarrollado infraestructura vial, asentamientos humanos, edificios públicos y privados que albergan actividades de gobierno, y centros educativos. Otra afectación inmediata se presenta en las vialidades que conectan la ciudad de este a oeste, y que intersectan con el Río Nuevo, pues se observó en los mapas de inundación una discontinuidad vial que divide a la ciudad en dos partes, dificultando la comunicación. Este fenómeno se presenta en cualquiera de los cuatro periodos de retorno analizados. Por otro lado, se propone llevar a cabo este análisis por microcuenca, para establecer el nivel de encharcamiento e inundación en diferentes partes de la ciudad.

Además, se propone analizar el comportamiento hidráulico del embovedado del Río Nuevo, integrándolo como parte de las variables de entrada del modelo, para optimizar los resultados obtenidos en HEC-RAS.

Por último, los resultados de esta investigación podrán ser utilizados por los planeadores urbanos, autoridades municipales, estatales, federales, sistemas de protección civil y la sociedad en general, a fin de planificar acciones que den soluciones integrales a la gestión de los riesgos asociados con inundaciones súbitas.

\section{Referencias}

Alaghmand, S., Abdullah, R., Abustan, I., \& Vosoogh, B. (2010). GISbased river flood hazard mapping in urban area (a case of study in Kayu Ara River Basin, Malaysia). International Journal of Engineering and Technology, 2(6), 488-500.

Dewan, T. (2015). Societal impacts and vulnerability to floods Blangladesh and Nepal. Weather and Climate Extremes, 7, 36-42. 
Heimhuber, V. (2013). GIS based flood modeling as part of an integrated development strategy for informal settlements (tesis de maestría). Technische Universität München, Múnich, Alemania.

Hernández-Uribe, R., Barrios-Piña, H., \& Ramírez, A. (2017). Análisis de riesgo por inundación: metodología y aplicación a la cuenca Atemajac. Tecnología y Ciencias del Agua, 8(3), 5-25.

Hosking, J. R. M, \& Wallis, J. R. (1997) Regional frequency analysis: An approach based on l-moments. Cambridge, UK: Cambridge University Press. Recuperado de http://dx.doi.org/10.1017/cbo9780511529443

Imperial County. (2013). Imperial County Multi-Jurisdiction Hazard Mitigation Plan Update. Recovered from http://www.co.imperial.ca.us/announcements\%5CPDFs\%5CImperi alCountyMHMPUpdate2013_121913.pdf

INEGI, Instituto de Estadística, Geografía e Informática. (2010). Imagen Lidar. México, DF, México: Instituto de Estadística, Geografía e Informática.

INEGI, Instituto de Estadística, Geografía e Informática. (2017). Simulador de Flujos de Agua de Cuencas Hidrográficas. Recuperado de http://antares.inegi.org.mx/analisis/red_hidro/siatl/\#

López, J. J., González, M., Scaini, A., Goñi, M., Valdenebro, J., \& Gimena, F. N. (2012). Caracterización del modelo HEC-HMS en la cuenca de río Arga en Pamplona y su aplicación a cinco avenidas significativas. Obras y Proyectos, 12, 15-30.

Magaña-Hernández, F., Bá, K., \& Guerra-Cobián, V. H. (2013). Estimación del hidrograma de crecientes con modelación determinística y precipitación derivada de radar. Agrociencia, 47, 739-752.

Maksimovic, C. (2001). Urban drainage in arid and semi-arid climates, 40(2). París, Francia: Organización de las Naciones Unidas para la Educación, la Ciencia y la Cultura, UNESCO.

Malekinezhad, H., \& Zare-Garizi, A. (2014). Regional frequency analysis of daily rainfall extremes using L-moments approach. Atmósfera, 27(4), 411-427.

Mulvany, T. J. (1850). On the use of self-registering rain and flood gauges. Proceedings of the Institution of Civil Engineers, 4(2), 1-8.

NRCS, Natural Resources Conservation Service. (1986). Technical release 55. Washington, DC, USA: U.S. Department of Agriculture.

Núñez-Cobo, J., Verbist, K., Ramírez-Hernández, J., \& Hallack-Alegría, M. (2010). Guía metodológica para la aplicación del análisis regional de frecuencia de sequias basado en L-momentos y resultados de 
aplicación en América latina. Montevideo, Uruguay: Organización de las Naciones Unidas para la Educación, la Ciencia y la Cultura, UNESCO.

Ouma, Y., \& Tateishi, R. (2014). Urban flood vulnerability and risk mapping using integrated multi-parametric AHP and GIS: Methodological overview and case study assessment. Water, 6(6), 2073-4441.

Parhi, P., Sankhua, R., \& Roy, G. (2012). Calibration of channel roughness for Mahanadi River (India), using HEC-RAS model. Journal of Water Resource and Protection, 4(10), 847-850.

Patel, K. (2009). Watershed modeling using HEC-RAS, HEC-HMS, and GIS models-A. New Jersey, USA: Rutgers, The State University of New Jersey.

Pourreza, B. M., Samadi, S. Z., Akhoond A. M., \& Ghahraman, A. B. (2016). Reliability of semiarid flash flood modeling. Journal of Hydrologic Engineering, 22(4), 05016039.

Rodríguez, S., González, P., Medina, N., Pardo, C., \& Santos, R. (2007). Propuesta Metodológica para la generación de mapas de inundación y clasificación de zonas de amenaza. Caso de estudio en la parte baja del Río Las Ceibas (Neiva-Hulia). Avances en Recursos Hidráulicos, 16, 65-78.

Rodríguez-Burgueño, J. E., Shanafield, M., \& Ramírez-Hernández, J. (2017). Comparison of infiltration rates in the dry river bed of the Colorado River Delta during environmental flows. Ecological Engineering, 106, 675-682.

Schaefer, M. R., Barker, B. L., Taylor, G. H., \& Wallis, J. R. (2006). Regional precipitation-frequency analysis and spatial mapping of precipitation for 24-hour and 2-hour durations in Eastern Washington. Washington, DC, USA: Washington State Department of Transportation.

Sivakumar, M. G., Das, H., \& Brunini, O. (2005). Impacts of present and future climate variability and change on agriculture and forestry in the arid and semi-arid tropics. Climate Change, 31-72. DOI 10.1007/1-4020-4166-7_4

The Salton Sea Authority. (2015). A brief description of its current conditions, and potential remediation projects. Recovered from http://www.sci.sdsu.edu/salton/SaltonSeaHomePage.html

Trivedi, A., Singh, A., \& Indian, A. (2015). Analysis of key factors for waste management in humanitarian response: An interpretive structural modelling approach. International Journal of Disaster Risk Reduction. DOI: 10.1016/j.ijdrr.2015.10.006 
UN-HABITAT, United Nations Human Settlements Programme (2011). UN-Habitat Annual Report 2010. Nairobi, Kenya: United Nations Human Settlements Programme.

US Department of the Interior Bureau of Reclamation. (2012). Colorado River Basin Water Supply and Demand Study. Washington, DC, USA: The U.S. Department of the Interior.

USACE, US Army Corps of Engineers. (2016). HEC-RAS River Analysis System User's Manual Version 5.0. US Army Corps of Engineers. Hydrologic Engineering Center. Washington, DC, USA: US Army Corps of Engineers.

Vargas-Castañeda, G., Ibáñez-Castillo, L. A., \& Arteaga-Ramírez, R. (2015). Development, classification and trends in rainfall-runoff modeling. Ingeniería Agrícola y Biosistemas, 7(1), 5-21. DOI: 10.5154/r.inagbi.2015.03.002

Wallis, J. R., Schaefer, M. G., Barker, B. L., \& Taylor, G. H. (2007). Regional precipitation- frequency analysis and spatial mapping for 24 hours and 2 hours durations for Washington State. Hydrology \& Earth System Sciences, 11(1), 415-442.

Wang, M., Zhang, L., \& Baddo, T. D. (2016). Hydrological modeling in a semi-arid region using HEC-HMS. Journal of Water Resource and Hydraulic Engineering, 105-115. Recuperado de http://dx.doi.org/10.4314/jfas.v9i2.27

Wolfs, V., Meert, P., Willems, P. (2015). Modular conceptual modelling approach and software for river hydraulic simulations. Environmental Modelling and Software, 71, 60-77. 